\title{
A novel role for CCR3 in brain T-cell infiltration and cognition in aging
}

\section{Arnaud Teichert}

Alkahest, Inc.

\section{Sanket Rege}

Alkahest, Inc. https://orcid.org/0000-0001-9145-1664

Juliet Masumi

Alkahest, Inc.

Lily Akrapongpisak

Alkahest, Inc.

Hannah Hackbart

Alkahest, Inc.

Onkar Dhande

Alkahest, Inc.

\section{Sofia Caryotakis}

Alkahest, Inc.

\section{Dino Leone}

Alkahest, Inc.

\section{Balazs Szoke}

Alkahest, Inc.

Jonas Hannestad

Alkahest, Inc.

Karoly Nikolich

Alkahest, Inc.

Steven Braithwaite

Alkahest, Inc.

S. Minami ( $\nabla$ sminami@alkahest.com )

Alkahest, Inc.

Keywords:

Posted Date: March 1st, 2021

DOl: https://doi.org/10.21203/rs.3.rs-247891/v1 
License: (c) (i) This work is licensed under a Creative Commons Attribution 4.0 International License. Read Full License 


\section{Abstract}

The authors have requested that this preprint be removed from Research Square. 